\title{
COVID-19 in Bulgarian Social Media: Factuality, Harmfulness, Propaganda, and Framing
}

\author{
Preslav Nakov, ${ }^{1}$ Firoj Alam, ${ }^{1}$ Shaden Shaar, ${ }^{1}$ \\ Giovanni Da San Martino ${ }^{2}$ and Yifan Zhang ${ }^{1}$ \\ ${ }^{1}$ Qatar Computing Research Institute, HBKU, Qatar \\ ${ }^{2}$ University of Padova, Italy \\ \{pnakov, fialam, sshaar, yzhang\}@hbku.edu.qa, \\ dasan@math.unipd.it
}

\begin{abstract}
With the emergence of the COVID-19 pandemic, the political and the medical aspects of disinformation merged as the problem got elevated to a whole new level to become the first global infodemic. Fighting this infodemic is currently ranked very high on the list of priorities of the World Health Organization, with dangers ranging from promoting fake cures, rumors, and conspiracy theories to spreading xenophobia and panic. With this in mind, we studied how COVID-19 is discussed in Bulgarian social media in terms of factuality, harmfulness, propaganda, and framing. We found that most Bulgarian tweets contain verifiable factual claims, are factually true, are of potential public interest, are not harmful, and are too trivial to fact-check; moreover, zooming into harmful tweets, we found that they spread not only rumors but also panic. We further analyzed articles shared in Bulgarian partisan pro/con-COVID-19 Facebook groups and found that propaganda is more prevalent in skeptical articles, which use doubt, flag waving, and slogans to convey their message; in contrast, concerned ones appeal to emotions, fear, and authority; moreover, skeptical articles frame the issue as one of quality of life, policy, legality, economy, and politics, while concerned articles focus on health \& safety.
\end{abstract}

\section{Introduction}

The ongoing global COVID-19 pandemic has brought an unprecedented situation with a lot of uncertainty: as this was a new disease, very little was known about it. This created an information void, where there was a lot of demand but little supply of reliable new information: a perfect breeding ground for all kinds of rumors and conspiracy theories, whose spread was facilitated by social media, which in turn optimized for user engagement (yet, later, they did put serious efforts in trying to limit the spread of false claims about COVID-19).
Unlike previous events that attracted a lot of disinformation, the emergence of the COVID-19 pandemic gave rise to a new powerful blending of medical and political disinformation, which resulted in the first global infodemic. Indeed, shortly after having declared the COVID-19 outbreak a pandemic, the World Health Organization had to engage in counter-measures against the growing infodemic, which it ranked among its top priorities in the fight against the COVID-19 pandemic. $^{1}$

Figure 1 shows some tweets that demonstrate how COVID-19 is discussed in Bulgarian social media. We can see that the problem goes beyond factuality: while some tweets spread rumors (Figure 1a), other discuss cure (Figure 1b). Indeed, the infodemic quickly extended to promoting bad cure, instilling panic, xenophobia, racism, and distrust in authorities, among others. (Alam et al., 2021b)

\section{I just saw Kрай на cnopoßeme за npouзхоgа на COVID-19! Tоŭ е uзkycmBeно съзgageн В САЩ nрез 2015 2. - Click to see also r thebulgariantimes.com/\%d0\%9a\%d1\%80\%d... \\ I just saw End of controversy over the origin of COVID-19! It was artificially created in USA in 2015 - Click to see also w- https://t.co/ikFXAmp8bO}

\section{(a) rumor}

Открито е първото лекарство, което доказано спасява от COVID-19 https://t.co/rUx9EAO5gx

The first drug that has been proven to save from COVID-19 has been found https://t.co/rUx9EAO5gx

\section{(b) discusses cure}

Figure 1: Bulgarian tweets with English translation.

\footnotetext{
${ }^{1}$ https: //www. who. int/health-topics/ infodemic
} 
Thus, it is important to analyze social media posts in terms of factuality, harmfulness, checkworthiness, etc. It is also useful to understand whether the post is propagandistic, what propaganda techniques are used, and how the issue is framed. While there have been studies focusing on (some of) these issues for high-resource languages such as English and Arabic (Barrón-Cedeño et al., 2020; Hossain et al., 2020; Li et al., 2020; Alam et al., 2021b; Nakov et al., 2021a,c), there has been less work for low-resource languages such as Bulgarian (Dinkov et al., 2019; Alam et al., 2021d; Shaar et al., 2021b,c). Here, we aim to bridge this gap by analyzing tweets and Facebook posts about COVID-19 in Bulgarian, with focus on factuality, harmfulness, propaganda, and framing.

Our contributions can be summarized as follows:

- We create a dataset of tweets and Facebook posts related to COVID-19. ${ }^{2}$

- We perform analysis from various perspectives (factuality, harmfulness, propaganda, and framing), and we discuss some interesting observations from our analysis.

The rest of the paper is organized as follows: Section 2 offers a brief overview of previous work. Section 3 describes the dataset. Section 4 discusses our methodology. Section 5 discusses the findings. Finally, Section 7 concludes and points to possible directions for future work.

\section{Related Work}

Below, we discuss work relevant to our analysis, focusing on factuality, check-worthiness, propaganda, framing, and fighting the COVID-19 infodemic.

\subsection{Factuality}

A variety of task formulations have been proposed to address the spread of misinformation and disinformation online, and for each formulation, a number of approaches have been developed. Some good readings on the topic include surveys such as that by Shu et al. (2017), who adopted a data mining perspective on "fake news" and focused on social media. Another survey (Zubiaga et al., 2018) studied rumor detection in social media. The survey by Thorne and Vlachos (2018) took a factchecking perspective on "fake news" and related problems.

\footnotetext{
2http://gitlab.com/sshaar/ covid-19-in-bulgarian-social-media
}

Li et al. (2016) covered truth discovery in general. Lazer et al. (2018) offered an overview and discussion on the science of "fake news". Vosoughi et al. (2018) focused on the proliferation of true and false news online. Other recent surveys focused on stance detection (Küçük and Can, 2020), propaganda (Nakov et al., 2021b), social bots (Ferrara et al., 2016), false information (Zannettou et al., 2019), and bias on the Web (Baeza-Yates, 2018). Some very recent surveys featured stance for misinformation and disinformation detection (Hardalov et al., 2021), automatic fact-checking to assist human fact-checkers (Nakov et al., 2021b), predicting the factuality and the bias of entire news outlets (Nakov et al., 2021d), and multimodal disinformation detection (Alam et al., 2021a).

A large body of research has focused on developing automatic systems for fact-checking to limit the spread of disinformation and misinformation ( $\mathrm{Li}$ et al., 2016; Hardalov et al., 2016; Shu et al., 2017; Lazer et al., 2018; Mihaylova et al., 2018; Vosoughi et al., 2018; Nguyen et al., 2020). This includes development of datasets (Wang, 2017; Augenstein et al., 2019), and organizing evaluation campaigns (Derczynski et al., 2017; Nakov et al., 2018; Da San Martino et al., 2019; Elsayed et al., 2019; Gorrell et al., 2019; Mihaylova et al., 2019; Barrón-Cedeño et al., 2020; Nakov et al., 2021c; Shaar et al., 2021b). However, there are credibility issues with automated systems (Arnold, 2020). Hence, another research direction has emerged: building tools to facilitate human fact-checkers (Nakov et al., 2021b).

\subsection{Check-Worthiness Estimation}

Most work on check-worthiness focused on political debates and speeches. This includes the ClaimBuster (Hassan et al., 2015) and the ClaimRank systems (Jaradat et al., 2018), shared tasks at CLEF (Atanasova et al., 2018, 2019; Shaar et al., 2020, 2021c), modeling the context of the claim (Gencheva et al., 2017; Patwari et al., 2017; Shaar et al., 2021a), and multi-task learning from the decisions of multiple fact-checking organizations (Vasileva et al., 2019).

There has been less research on identifying check-worthy claims in social media posts. Previous work in this direction includes checkworthiness estimation of COVID-19 and political tweets (Alam et al., 2021d,b; Shaar et al., 2020, 2021b,c). 
More directly related to our work here is the work of Alam et al. (2021d) and Alam et al. (2021b), who developed a multi-question annotation schema to annotate tweets about COVID-19, organized around seven questions that model the perspective of journalists, fact-checkers, social media platforms, policymakers, and the society. In our experiments, we use their schema and data to train classifiers for part of our analysis.

\subsection{Propaganda}

Propaganda is a communication tool, deliberately designed to influence the opinions and the actions of other people in order to achieve a predetermined goal. Computational propaganda is defined as the use of automated approaches to intentionally disseminate misleading information on social media platforms (Woolley and Howard, 2018).

Most research on propaganda detection has focused on analyzing textual content (Barrón-Cedeno et al., 2019; Rashkin et al., 2017; Da San Martino et al., 2019; Da San Martino et al., 2019, 2020a). Rashkin et al. (2017) developed the TSHP-17 corpus, which uses document-level annotation and is labeled with four classes: trusted, satire, hoax, and propaganda. They trained a model using word $n$-gram representation with logistic regression and reported that the model performed well only on articles from sources that the system was trained on. Barrón-Cedeno et al. (2019) developed the QP rop corpus with two labels: propaganda vs. non-propaganda. They also experimented on TSHP-17 and QP rop corpora, where for the TSHP -17 corpus, they binarized the labels: propaganda vs. any of the other three categories. Similarly, Habernal et al. $(2017,2018)$ developed a corpus with $1.3 \mathrm{k}$ arguments annotated with five fallacies, including ad hominem, red herring, and irrelevant authority, which directly relate to propaganda techniques.

A more fine-grained propaganda analysis was done by Da San Martino et al. (2019), who developed a corpus of news articles annotated with 18 propaganda techniques. Subsequently, the Prta system was released (Da San Martino et al., 2020b), and improved models were proposed, focusing on interpretability (Yu et al., 2021) or addressing the limitations of transformers (Chernyavskiy et al., 2021). Very recently, multimodal content was explored in memes using 22 fine-grained propaganda techniques (Dimitrov et al., 2021a,b).

\subsection{Framing}

Framing is a strategic device and a central concept in political communication, for representing different salient aspects and perspectives for the purpose of conveying the latent meaning about an issue (Entman, 1993). It is important for news media as the same topics can be discussed from different perspectives, which can influence our understanding, beliefs, and attitudes regarding what is happening in our society. There has been recent work on automatically identifying media frames, which includes developing coding schemes and datasets such as the Media Frames Corpus (Card et al., 2015), developing systems to automatically detect media frames (Liu et al., 2019; Zhang et al., 2019), large scale automatic analysis of New York Times Articles (Kwak et al., 2020), and a semisupervised approach to detecting frames in online news sources (Cheeks et al., 2020).

\subsection{COVID-19 Research}

Since the beginning of the COVID-19 pandemic, there has been a large number of work on fighting the COVID-19 infodemic. Most notable work includes developing multi-question annotation schemas of tweets about COVID-19 (Alam et al., 2021d,b), studying credibility (Cinelli et al., 2020; Pulido et al., 2020; Zhou et al., 2020), racial prejudices and fear (Medford et al., 2020; Vidgen et al., 2020), situational information, e.g., caution and advice (Li et al., 2020), as well as on detecting mentions and stance with respect to known misconceptions (Hossain et al., 2020).

Another less relevant research line is on the development of datasets of tweets about COVID-19 (Cinelli et al., 2020; Song et al., 2021; Zhou et al., 2020; Haouari et al., 2021)

\section{Dataset}

Tweets: Using the Twitter API, we collected 30k tweets from January 2020 till November 2020. We performed search by specifying the target language to be Bulgarian and asking for the tweet to contain the following keywords and hashtags related to COVID-19 (English translations are shown in bleu color):

\#корона, \#коронавирус, коронавирус, корона \#corona, \#coronavirus, coronavirus, corona 


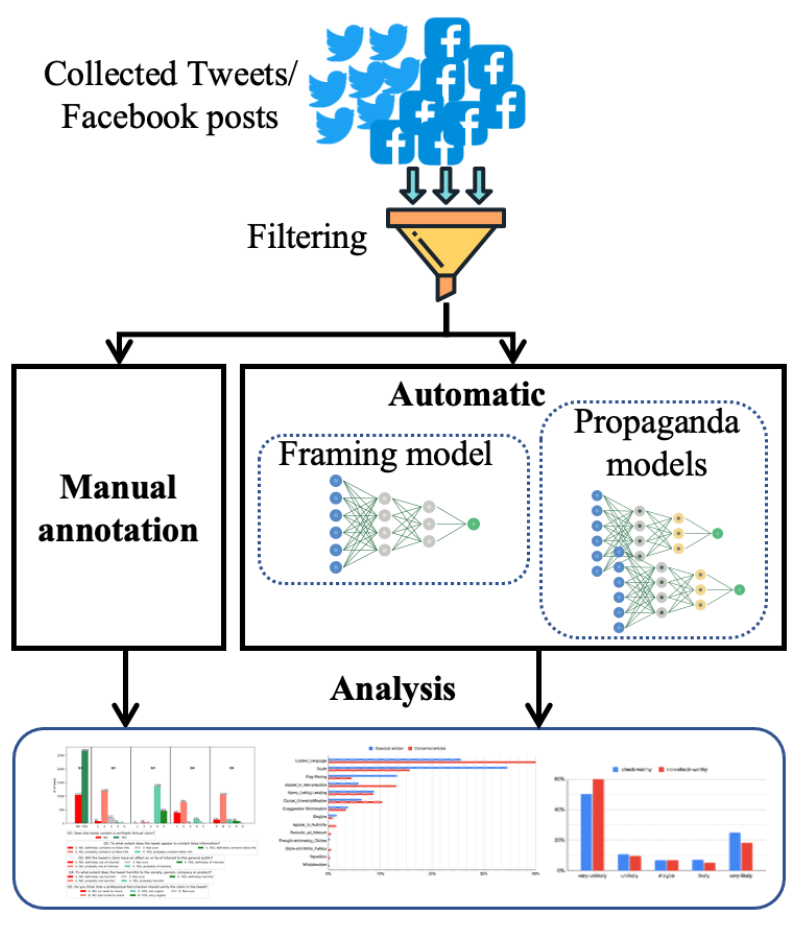

Figure 2: The system architecture of our analysis. The arrows indicate the information flow.

We only selected original tweets (no retweets or replies), we removed duplicates using a similaritybased approach (Alam et al., 2021c), and we filtered out tweets with less than five words. Finally, we selected the most frequently liked and retweeted tweets for annotation. For our analysis, we manually annotated $4 \mathrm{k}$ of them using the multi-question annotation schema from (Alam et al., 2021b), with three annotators per tweet (a total of $11 \mathrm{k}$ annotations). This Bulgarian data is also used in (Alam et al., 2021d) and for the CLEF 2021 CheckThat! lab task 1 (Shaar et al., 2021c).

Articles in Facebook posts: We further collected articles posted in Bulgarian Facebook groups that discuss COVID-19. We focused on concerned, skeptical, and conspiracy groups; the list is shown in Figure 3. We collected the links to articles posted in these groups, and we manually annotated each article as skeptical or concerned.

\section{Method}

Figure 2 shows our analysis pipeline. Below, we discuss each element of the pipeline in more detail.

\subsection{Manual Annotation}

The manual tasks consist of multi-question disinformation annotation of tweets and also of skeptical vs. concerned articles posted on Facebook.

\begin{tabular}{|c|}
\hline Concerned \\
\hline $\begin{array}{l}\text { (10.9k) Covid-19: факти срещу слухове } \\
\text { (10.9k) Covid-19: facts against rumors } \\
\text { (2.4k) Коронавирус/COVID-19 - само } \\
\text { валидирана информация } \\
\text { (2.4k) Coronavirus / COVID-19 - } \\
\text { validated information only } \\
\text { (1.0k) Здрав разум за здрава държава } \\
\text { (1.0k) Common sense for a healthy state }\end{array}$ \\
\hline Skeptical \\
\hline $\begin{array}{l}\text { (14.8k) Аз подкрепям доцент Мангъров } \\
\text { (14.8k) I support Associate Professor } \\
\text { Mangarov } \\
\text { (6.5k) Подкрепа за Атанас Мангъров } \\
\text { (6.5k) Support for Atanas Mangarov } \\
\text { (4.0k) С доц. д-р Манг*р*в и Бог } \\
\text { обратно в живота } \\
\text { (4.0k) With Assoc. Prof. Dr. Mang * } r * v \\
\text { and God back to life }\end{array}$ \\
\hline Conspiracy \\
\hline $\begin{array}{l}\text { (1.4k) ИСТИНАТА : Коронавирус / } \\
\text { COVID-19, КОВИД-19/ } \\
\text { (1.4k) ТНЕ ТRUТН: Coronavirus / } \\
\text { COVID-19, СОVID-19/ } \\
\text { (1.3k) Измислицата Корона- вирус } \\
\text { (1.3k) The Corona virus } \\
\text { (1.2k) Измамата Ковид19 } \\
\text { (1.2k) The Kovid Deception19 } \\
\text { (0.2k) Корона вирус COVID-19 или } \\
\text { голямата манипулация } \\
\text { (0.2k) Crown virus COVID-19 or таjor } \\
\text { manipulation }\end{array}$ \\
\hline
\end{tabular}

Figure 3: Facebook groups we collected articles from.

\subsubsection{Disinformation Annotation for Tweets}

For the disinformation analysis, we used the holistic approach in (Alam et al., 2021b). It is formulated into seven questions, asking whether a tweet (Q1) contains a verifiable factual claim, (Q2) is likely to contain false information, (Q3) is of interest to the general public, (Q4) is potentially harmful to a person, a company, a product, or society, (Q5) requires verification by a fact-checker, (Q6) poses harm to society and why, or (Q7) requires the attention of policy makers and why. Three annotators worked on each tweet, following the annotation guidelines in (Alam et al., 2021b). 
The annotators were fluent in Bulgarian, two were male and one was female, with qualifications ranging from undergrad students to people with a MSc degree. For disagreed annotations, a final consolidator participated in the discussion to decide the final label. We computed the inter-annotator agreement between the annotators and the final consolidated label using Fleiss Kappa $(\kappa)$ as shown in Table 1. We can see that there was moderate to substantial agreement between the human annotators across the questions, according to the range of values for $\kappa$ suggested in (Landis and Koch, 1977).

\begin{tabular}{lccccccc}
\hline Agree. Pair & Q1 & Q2 & Q3 & Q4 & Q5 & Q6 & Q7 \\
\hline A1 - C & 0.77 & 0.44 & 0.64 & 0.53 & 0.49 & 0.53 & 0.51 \\
A2 - C & 0.51 & 0.40 & 0.59 & 0.49 & 0.44 & 0.56 & 0.53 \\
A3 - C & 0.47 & 0.38 & 0.57 & 0.49 & 0.38 & 0.53 & 0.40 \\
Avg & $\mathbf{0 . 5 8}$ & $\mathbf{0 . 4 1}$ & $\mathbf{0 . 6 0}$ & $\mathbf{0 . 5 0}$ & $\mathbf{0 . 4 4}$ & $\mathbf{0 . 5 4}$ & $\mathbf{0 . 4 8}$ \\
\hline
\end{tabular}

Table 1: Inter-annotator agreement using Fleiss Kappa for the 7-level annotation for disinformation in tweets.

\subsubsection{Skeptical vs. Concerned Annotation for Articles Posted on Facebook}

The same annotators further annotated the Facebook articles as skeptical or concerned. This was a fairly straightforward task, with almost no disagreement. Note that we analyzed each article manually to decide whether it is skeptical or concerned (rather than using distant supervision to propagate the label for the group to label articles automatically, even though teh vast majority of articles could be labeled with the label of the group).

\subsection{Automatic Classification}

For the analysis of propaganda and framing, both for tweets and for news articles, we used the automatic models discussed below.

\subsubsection{Propaganda Analysis}

For this analysis, we used Proppy and Prta.

Proppy (Barrón-Cedeño et al., 2019) uses is trained on $51 \mathrm{k}$ articles, and uses a maximum entropy model with various style-related features, such as character $n$-grams and a number of vocabulary richness and readability measures. The model achieves and F1 score of 82.89, as evaluated on a separate test set of $10 \mathrm{k}$ articles. It outputs the following propaganda labels based on the output score $p \in[0,1]$ : very unlikely $(0.0 \leq p<0.2)$, unlikely $(0.2 \leq p<0.4)$, somehow $(0.4 \leq p<0.6)$, likely $(0.6 \leq p<0.8)$, and very likely $(0.8 \leq p \leq 1.0)$.
The Prta system (Da San Martino et al., 2020b) offers a fragment-level and a sentence-level classifiers. They were trained on a corpus of $350 \mathrm{~K}$ tokens. The performance of the sentence-level classifier is 60.71 in terms of F1 score. The fragment-level classifier identifies the text fragments and the propaganda techniques that occur in them. They consider the following 18 techniques: (i) Loaded language, (ii) Name calling or labeling, (iii) Repetition, (iv) Exaggeration or minimization, (v) Doubt, (vi) Appeal to fear/prejudice, (vii) Flagwaving, (viii) Causal oversimplification, (ix) Slogans, $(x)$ Appeal to authority, (xi) Black-and-white fallacy, dictatorship, (xii) Thought-terminating cliché, (xiii) Whataboutism, (xiv) Reductio ad Hitlerum, (xv) Red herring, (xvi) Bandwagon, (xvii) Obfuscation, intentional vagueness, confusion, and (xviii) Straw man.

Note that both Proppy and Prta only support English. To prepare their input, we translated the Bulgarian text to English using Google.

\subsubsection{Framing}

We used the Tanbih Framing Bias Detection system (Zhang et al., 2019), trained on the Media Frames Corpus (11k training news articles) by finetuning BERT to detect topic-agnostic media frames, achieving accuracy of $66.7 \%$ on the test set $(1,138$ news articles). It can predict the following 15 frames: (i) Economy, (ii) Capacity and resources, (iii) Morality, (iv) Fairness and equality, (v) Legality, constitutionality and jurisprudence, (vi) Policy prescription and evaluation, (vii) Crime and punishment, (viii) Security and defense, (ix) Health and safety, (x) Quality of life, (xi) Cultural identity, (xii) Public opinion, (xiii) Politics, (xiv) External regulation and reputation, and (xv) Other.

\section{Results and Discussion}

Below, we present the results of our analysis.

\subsection{Disinformation Analysis}

Figure 4 shows a detailed distribution for each question. We can see that (i) most tweets contain a verifiable factual claim, (ii) about half of the tweets are factually true, (iii) most of them are of general interest to the public, (iv) about half of the tweets are not harmful to the society, to a person, a company, or a product, $(v)$ many tweets are trivial to fact-check, (vi) some tweets spread rumors, panic, or make a joke. 


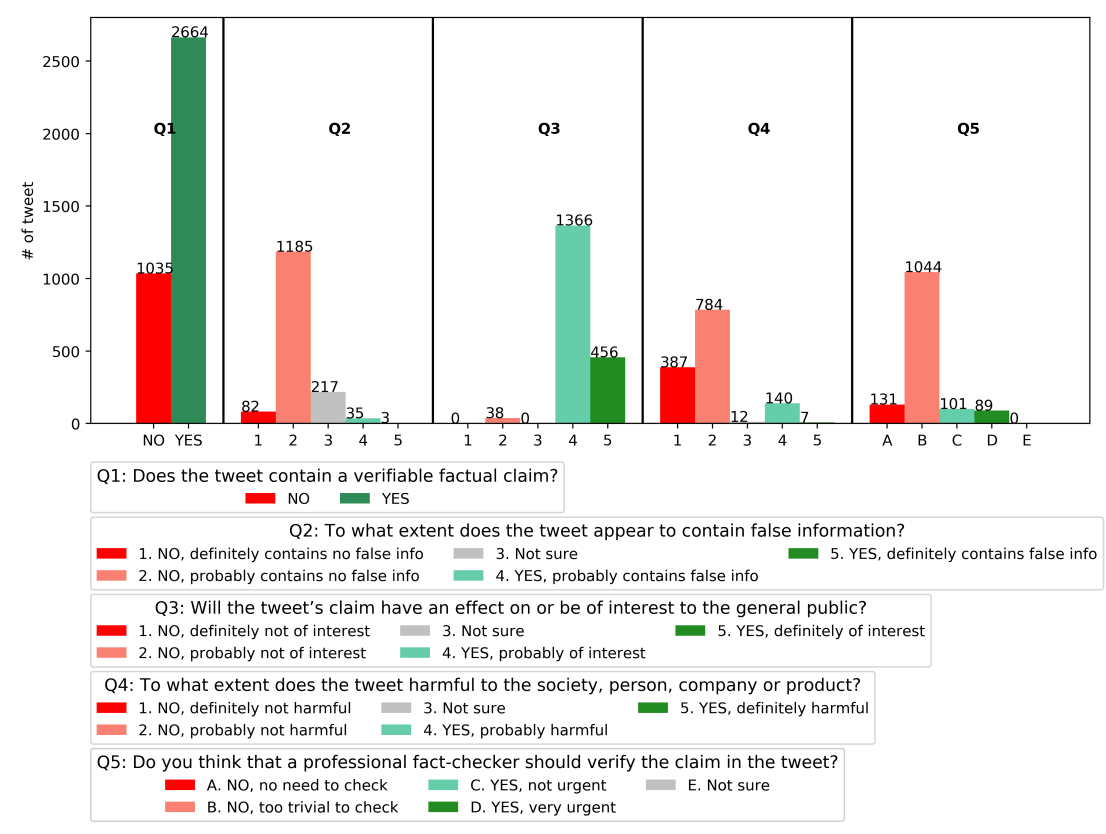

(a) Questions Q1-Q5.

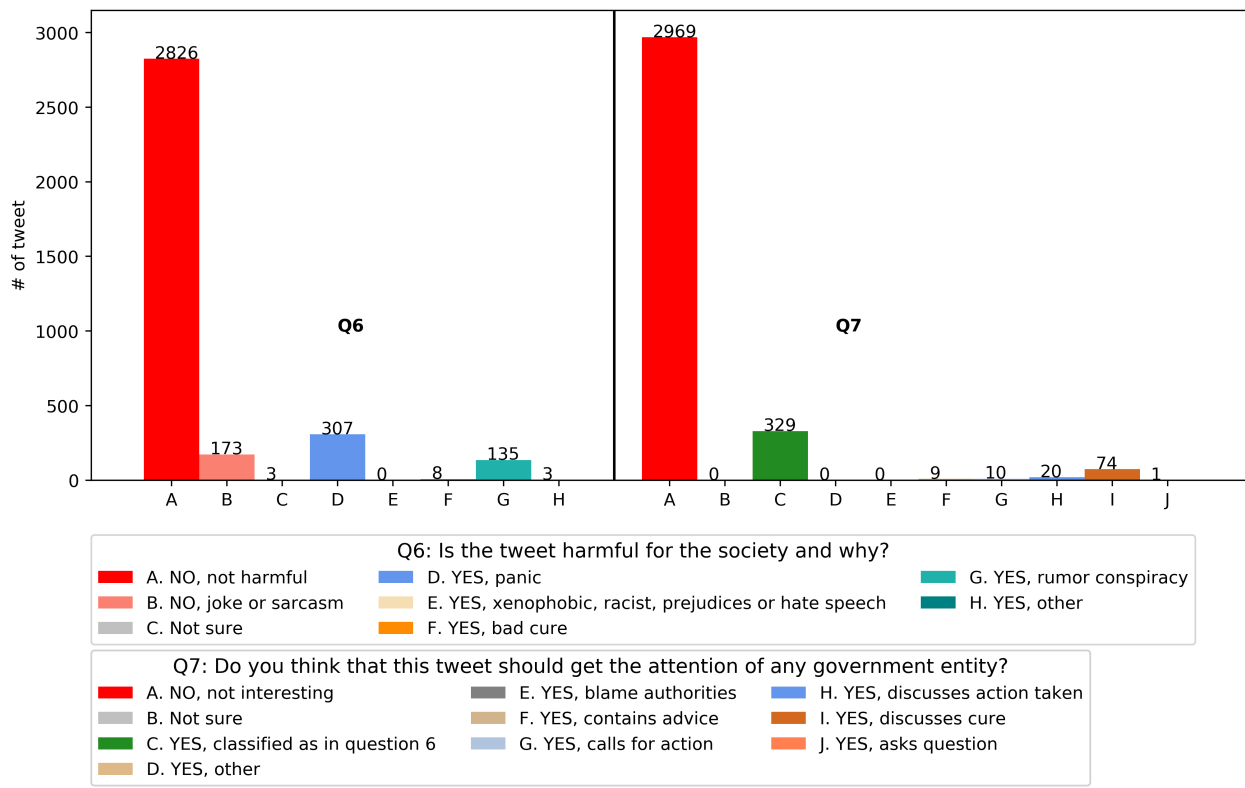

(b) Questions (Q6-7).

Figure 4: Statistics about the distribution of Bulgarian tweets from January to November 2020 (manually labeled).

\subsection{Propaganda Analysis}

Propaganda Figure 5 shows the results for the propaganda analysis of tweets associated with check-worthiness and harmfulness. We can see that check-worthy tweets are more propagandistic (right-side bars in Figure 5a). A large portion of them (left-side bars) are neither check-worthy nor propagandistic. On Figure 5b, we can see that harmful tweets (i.e., such spreading rumors, conspiracy, and panic) are (somewhat) more propagandistic than non-harmful ones.
Figure 5c shows the propaganda analysis for articles posted in Facebook groups. We can see that skeptical articles are more propagandistic.

Propaganda Techniques A more fine-grained analysis is important in order to understand the type of content that is shared/posted in social media. Thus, we analyzed tweets by categorizing them using propaganda techniques. Figure 6 shows a propaganda technique analysis for the tweets, which are also labeled for check-worthiness and harmfulness. 


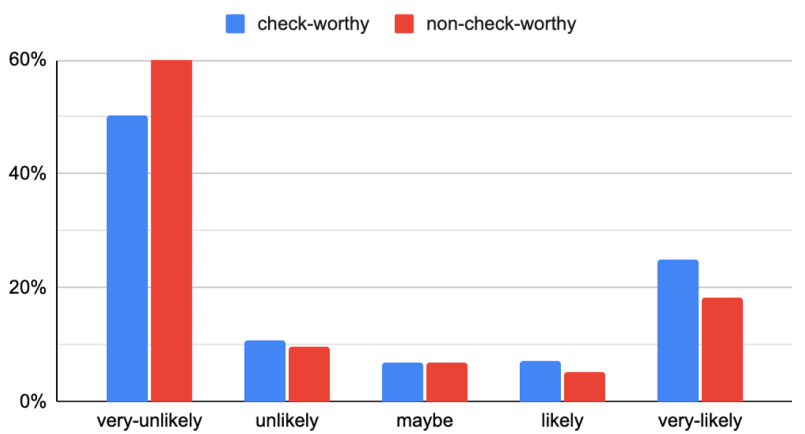

(a) Check-worthiness and propaganda in tweets. non-harmful rumor/conspiracy panic

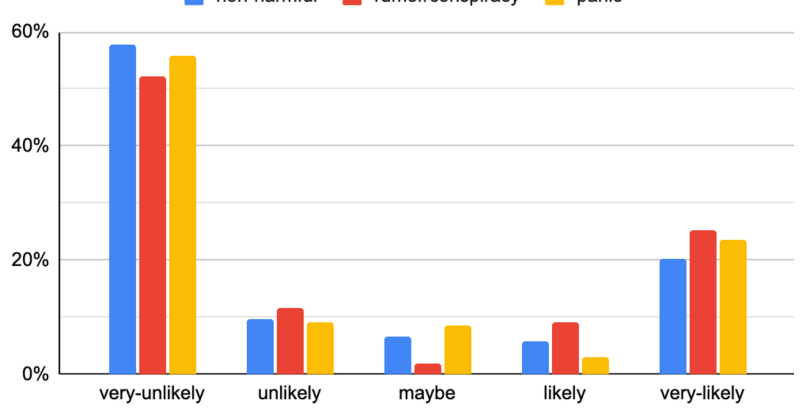

(b) Harmfulness and propaganda in tweets. $\square$ skeptical $\square$ concerned

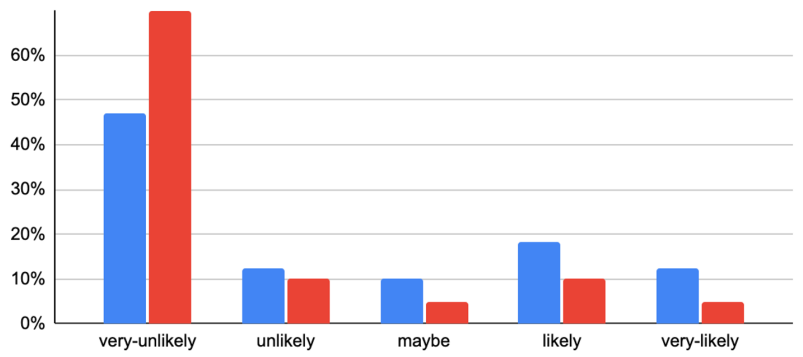

(c) Propaganda for skeptical vs. concerned articles posted in Facebook groups.

Figure 5: Propaganda.

Figure 6a shows that a higher proportion of check-worthy tweets are associated with fear, doubt, and loaded language, whereas non-checkworthy tweets are associated with exaggeration, flag-waving, name-calling, slogans, causal oversimplification, and repetition.

Figure $6 \mathrm{~b}$ further shows that check-worthy tweets are associated with name-calling; rumor and conspiracy tweets are associated with $d o u b t$; and panic tweets are associated with fear, exaggeration, loaded language, and flag-waving.

Figure $5 \mathrm{c}$ shows the distribution of the propaganda techniques in skeptical vs. concerned articles posted in Facebook groups. We can see that skeptical articles are associated with doubt, flag-waving, and slogans, whereas concerned articles use loaded language, appeal to fear, appeal to authority, and causal oversimplification.

\subsection{Framing}

Our analysis of framing in tweets shows that economy is the dominant perspective, health and safety come second, and legalilty is third. Figure 7 reports the distribution of tweets manually annotated for check-worthiness and harmfulness and automatically analyzed for framing. Figure 7 a shows that the most frequent check-worthy tweets are associated with health, legality, crime and punishment, whereas non-check-worthy are associated with economy, politics, and quality of life. Figure $7 \mathrm{~b}$ reports the distribution of the framing and the harmfulness labels. Frames labeled as economy are non-harmful; cultural identity, crime and punishment are associated with rumor/conspiracy, while health and safety frames show panic.

Figure $7 \mathrm{c}$ reports the distribution of articles manually categorized as skeptical vs. concerned and automatically analyzed for framing. The plot shows that skeptical articles are associated with quality of life, policy, legality, economy, and politics, whereas concerned articles are associated with health and safety, and cultural identity.

\section{Limitations}

Manual annotations Our manual annotation for disinformation in tweets shows moderate to substantial agreement across the questions. We believe that this is reasonable given the complexity of the task.

Automatic analysis The performance of the automatic analysis varies across the different tasks (e.g., for propaganda analysis vs. framing), which can introduce noise in the results.

Translation We needed to translate the text from Bulgarian to English, which can add noise in case of translation errors. Although we performed a qualitative analysis on a sample of propaganda annotations and we found a good quality for our model's predictions, in future work, we would like to train a model directly for Bulgarian.

\section{Conclusion and Future Work}

We presented our analysis of COVID-19 in Bulgarian social media with focus on tweets and on news articles posted in Facebook groups, which we collected in different time frames starting from January till November 2020. Then, we manually and automatically analyzed them using different aspects of disinformation, propaganda, and framing. 


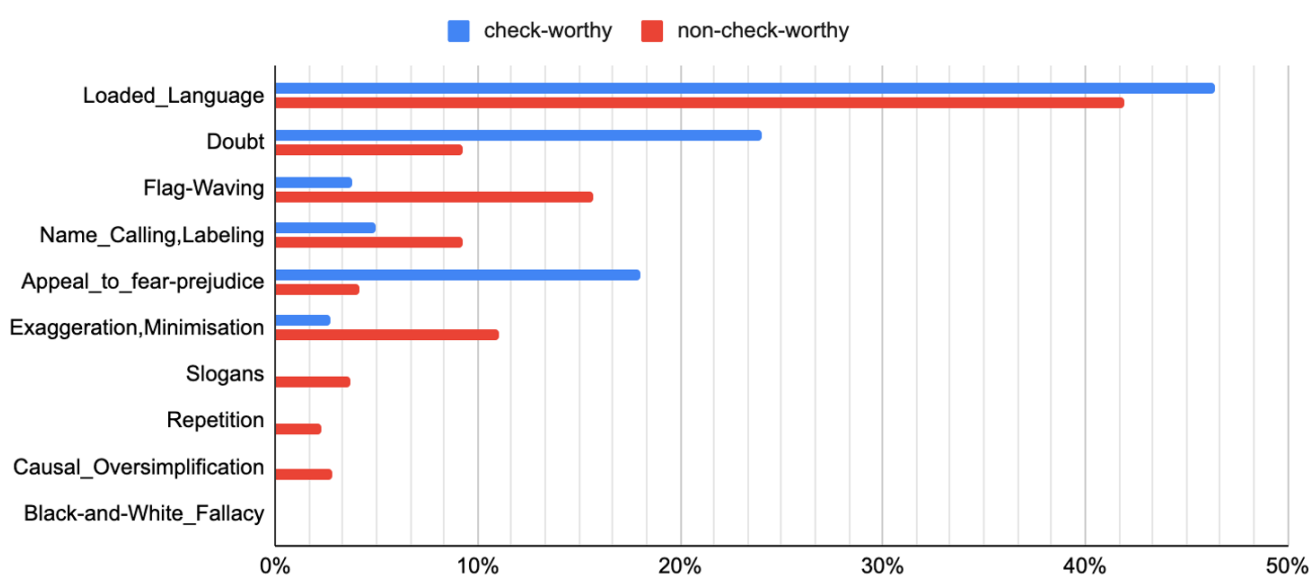

(a) Check-worthiness and propaganda techniques in tweets.

non-harmful rumors/conspiracy panic

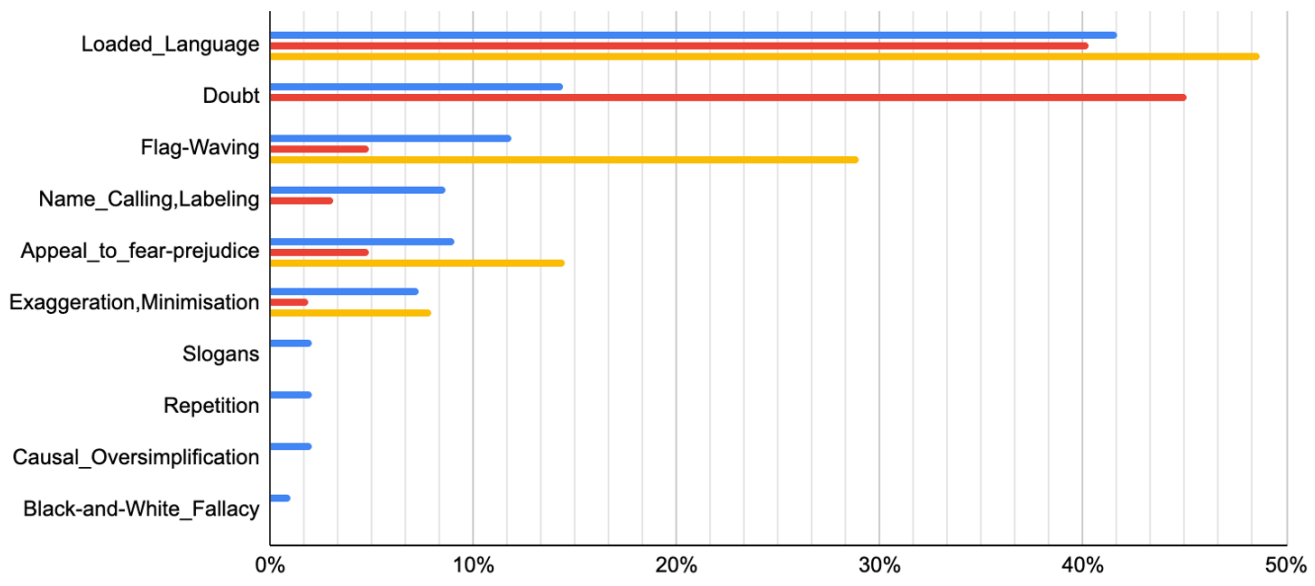

(b) Harmfulness and propaganda techniques in tweets.

Skeptical articles concerned articles

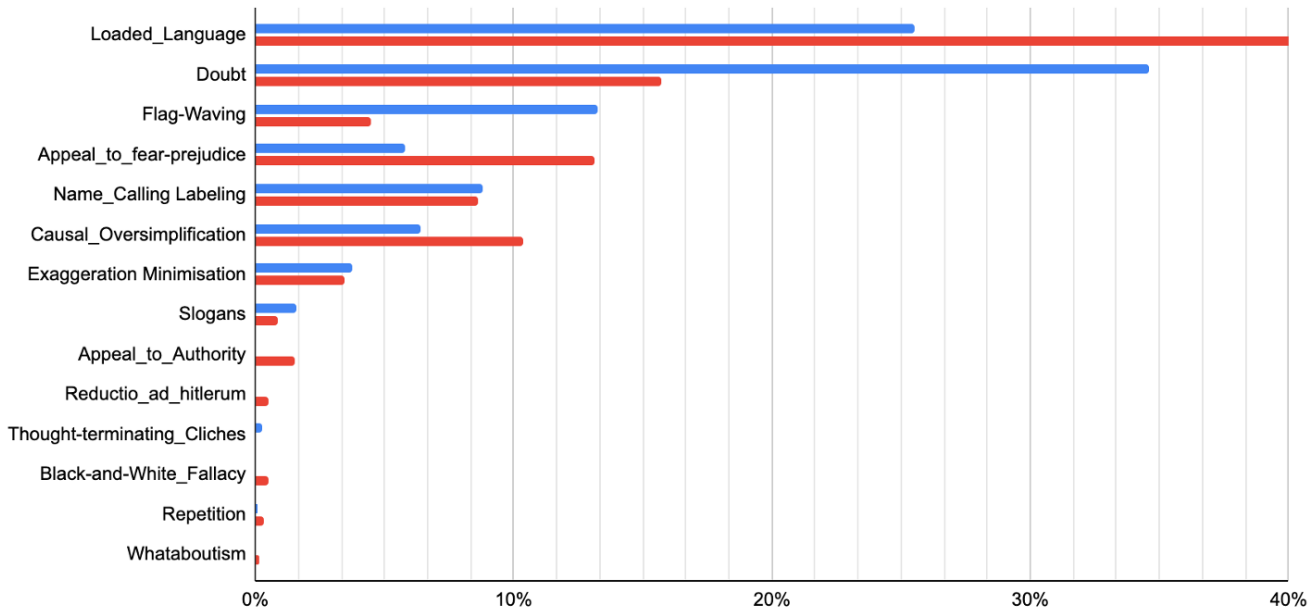

(c) Propaganda techniques for skeptical vs. concerned articles posted in Facebook groups.

Figure 6: Propaganda techniques.

We believe that the kind of analysis we perform here would help in better understanding various trends in social media about COVID-19. See also a related study about COVID-19 and vaccines in Qatar (Nakov et al., 2021a).
There are a number of interesting research directions that could be pursued using the approaches we used in this study. While we only focused on Twitter and Facebook, similar analysis can be done on other platforms e.g., WhatsApp, Gab, Reddit. 


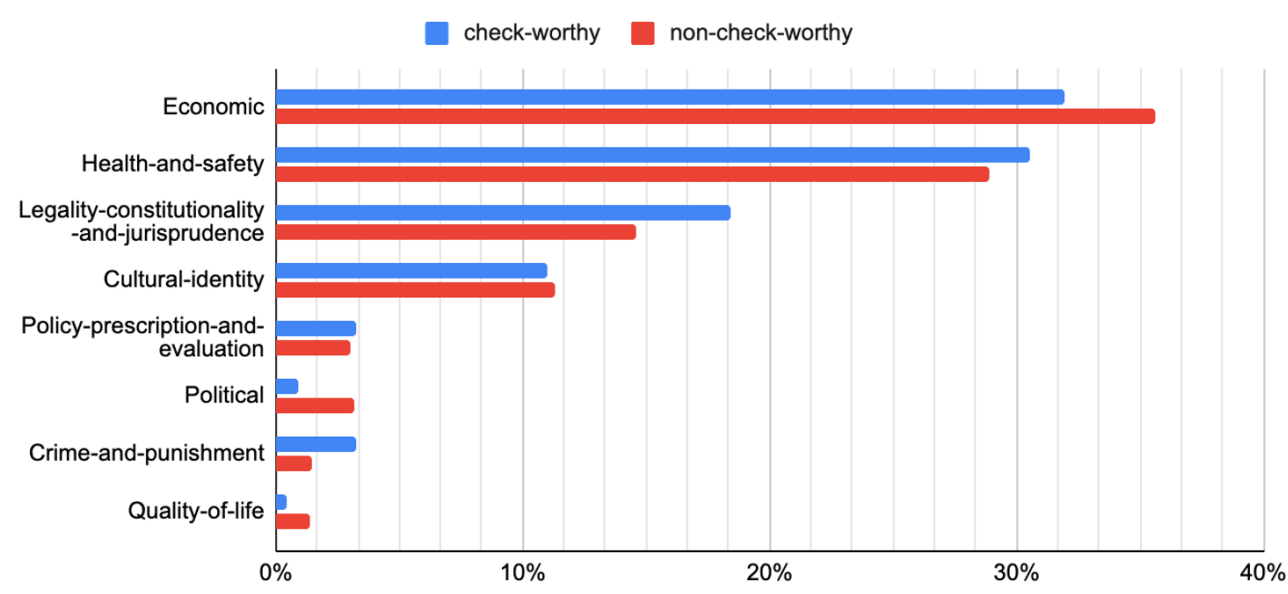

(a) Check-worthiness and framing in tweets.

non-harmful rumor/conspiracy panic

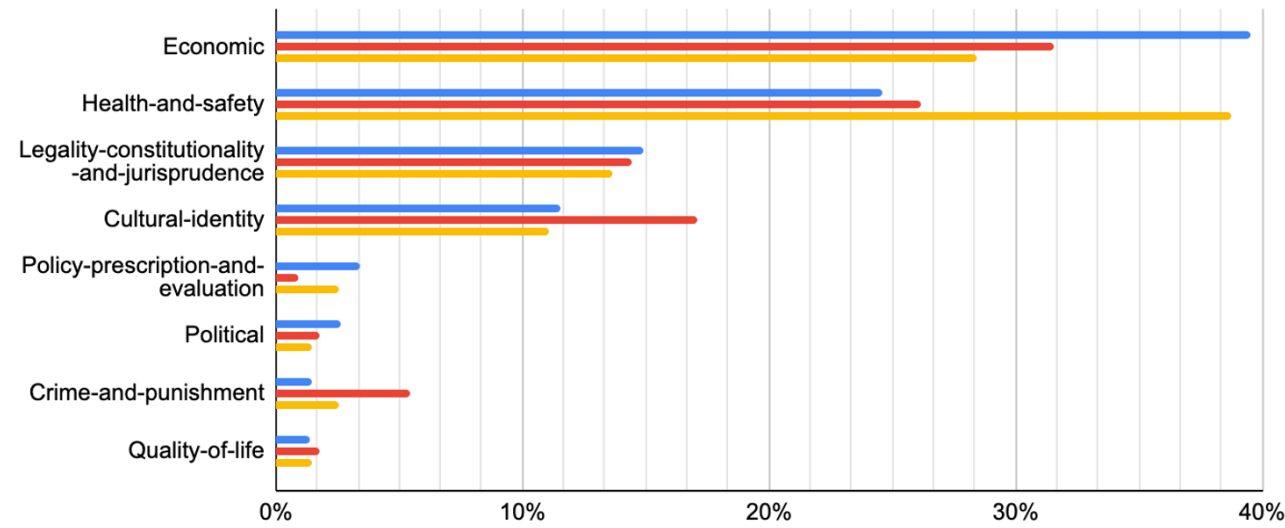

(b) Harmfulness and framing in tweets.

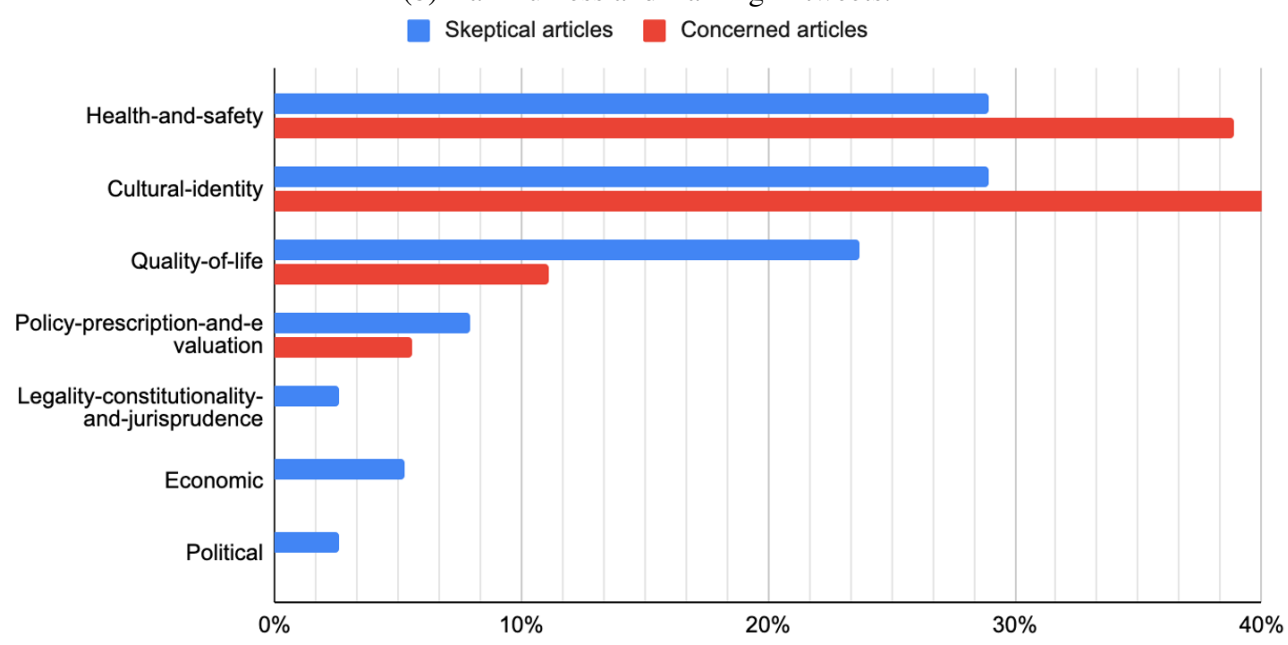

(c) Framing for skeptical vs. concerned articles posted in Facebook groups.

Figure 7: Framing.

\section{Acknowledgments}

We would like to thank the Atlantic Club in Bulgaria and DataBee for helping us with the manual analysis of Bulgarian tweets and of news articles posted on Facebook. We further thank the anonymous reviewers for their constructive comments.
This research is part of the Tanbih megaproject (http://tanbih.qcri.org), developed at the Qatar Computing Research Institute, HBKU, which aims to limit the impact of "fake news", propaganda, and media bias by making users aware of what they are reading, thus promoting media literacy and critical thinking. 


\section{References}

Firoj Alam, Stefano Cresci, Tanmoy Chakraborty, Fabrizio Silvestri, Dimiter Dimitrov, Giovanni Da San Martino, Shaden Shaar, Hamed Firooz, and Preslav Nakov. 2021a. A survey on multimodal disinformation detection. arXiv/2103.12541.

Firoj Alam, Fahim Dalvi, Shaden Shaar, Nadir Durrani, Hamdy Mubarak, Alex Nikolov, Giovanni Da San Martino, Ahmed Abdelali, Hassan Sajjad, Kareem Darwish, and Preslav Nakov. 2021b. Fighting the COVID-19 infodemic in social media: A holistic perspective and a call to arms. In Proceedings of the International AAAI Conference on Web and Social Media, ICWSM '21, pages 913-922.

Firoj Alam, Hassan Sajjad, Muhammad Imran, and Ferda Ofli. 2021c. CrisisBench: Benchmarking crisis-related social media datasets for humanitarian information processing. In Proceedings of the International AAAI Conference on Web and Social Media, ICWSM '21, pages 923-932.

Firoj Alam, Shaden Shaar, Fahim Dalvi, Hassan Sajjad, Alex Nikolov, Hamdy Mubarak, Giovanni Da San Martino, Ahmed Abdelali, Nadir Durrani, Kareem Darwish, Abdulaziz Al-Homaid, Wajdi Zaghouani, Tommaso Caselli, Gijs Danoe, Friso Stolk, Britt Bruntink, and Preslav Nakov. 2021d. Fighting the COVID-19 infodemic: Modeling the perspective of journalists, fact-checkers, social media platforms, policy makers, and the society. In Findings of EMNLP 2021.

Phoebe Arnold. 2020. The challenges of online fact checking. Technical report, Full Fact.

Pepa Atanasova, Lluís Màrquez, Alberto BarrónCedeño, Tamer Elsayed, Reem Suwaileh, Wajdi Zaghouani, Spas Kyuchukov, Giovanni Da San Martino, and Preslav Nakov. 2018. Overview of the CLEF-2018 CheckThat! Lab on automatic identification and verification of political claims, Task 1: Check-worthiness. In CLEF 2018 Working Notes, Avignon, France. CEUR-WS.org.

Pepa Atanasova, Preslav Nakov, Georgi Karadzhov, Mitra Mohtarami, and Giovanni Da San Martino. 2019. Overview of the CLEF-2019 CheckThat! Lab on Automatic Identification and Verification of Claims. Task 1: Check-Worthiness. In CLEF 2019 Working Notes, Lugano, Switzerland. CEURWS.org.

Isabelle Augenstein, Christina Lioma, Dongsheng Wang, Lucas Chaves Lima, Casper Hansen, Christian Hansen, and Jakob Grue Simonsen. 2019. MultiFC: A real-world multi-domain dataset for evidence-based fact checking of claims. In Proceedings of the Conference on Empirical Methods in Natural Language Processing and the 9th International Joint Conference on Natural Language Processing, EMNLP-IJCNLP '19, pages 4685-4697, Hong Kong, China.
Ricardo Baeza-Yates. 2018. Bias on the web. Commun. ACM, 61(6):54-61.

Alberto Barrón-Cedeño, Giovanni Da San Martino, Israa Jaradat, and Preslav Nakov. 2019. Proppy: A system to unmask propaganda in online news. In Proceedings of the Thirty-Third AAAI Conference on Artificial Intelligence, AAAI'19, pages 9847-9848, Honolulu, HI, USA.

Alberto Barrón-Cedeño, Tamer Elsayed, Preslav Nakov, Giovanni Da San Martino, Maram Hasanain, Reem Suwaileh, and Fatima Haouari. 2020. CheckThat! at CLEF 2020: Enabling the automatic identification and verification of claims in social media. In Proceedings of the 42nd European Conference on Information Retrieval, ECIR '19, pages 499-507, Lisbon, Portugal.

Alberto Barrón-Cedeno, Israa Jaradat, Giovanni Da San Martino, and Preslav Nakov. 2019. Proppy: Organizing the news based on their propagandistic content. Information Processing \& Management, 56(5):1849-1864.

Dallas Card, Amber E. Boydstun, Justin H. Gross, Philip Resnik, and Noah A. Smith. 2015. The Media Frames Corpus: Annotations of frames across issues. In Proceedings of the 53rd Annual Meeting of the Association for Computational Linguistics and the 7th International Joint Conference on Natural Language Processing, ACL-IJCNLP' 15, pages 438-444, Beijing, China.

Loretta H Cheeks, Tracy L Stepien, Dara M Wald, and Ashraf Gaffar. 2020. Discovering news frames: An approach for exploring text, content, and concepts in online news sources. In Cognitive Analytics: Concepts, Methodologies, Tools, and Applications, pages 702-721. IGI Global.

Anton Chernyavskiy, Dmitry Ilvovsky, and Preslav Nakov. 2021. Transformers: "The end of history" for NLP? In Proceedings of the European Conference on Machine Learning and Principles and Practice of Knowledge Discovery in Databases, ECMLPKDD'21.

Matteo Cinelli, Walter Quattrociocchi, Alessandro Galeazzi, Carlo Michele Valensise, Emanuele Brugnoli, Ana Lucia Schmidt, Paola Zola, Fabiana Zollo, and Antonio Scala. 2020. The COVID-19 social media infodemic. Sci Rep., 10:16598.

Giovanni Da San Martino, Alberto Barron-Cedeno, and Preslav Nakov. 2019. Findings of the NLP4IF-2019 shared task on fine-grained propaganda detection. In Proceedings of the 2nd Workshop on NLP for Internet Freedom (NLP4IF): Censorship, Disinformation, and Propaganda, NLP4IF '19, pages 162-170, Hong Kong, China.

Giovanni Da San Martino, Stefano Cresci, Alberto Barrón-Cedeño, Seunghak Yu, Roberto Di Pietro, and Preslav Nakov. 2020a. A survey on computational propaganda detection. In Proceedings of the 
Twenty-Ninth International Joint Conference on Artificial Intelligence, IJCAI '20, pages 4826-4832.

Giovanni Da San Martino, Shaden Shaar, Yifan Zhang, Seunghak Yu, Alberto Barrón-Cedeno, and Preslav Nakov. 2020b. Prta: A system to support the analysis of propaganda techniques in the news. In Proceedings of the Annual Meeting of Association for Computational Linguistics, ACL '20, pages 287293.

Giovanni Da San Martino, Seunghak Yu, Alberto Barrón-Cedeño, Rostislav Petrov, and Preslav Nakov. 2019. Fine-grained analysis of propaganda in news articles. In Proceedings of the 2019 Conference on Empirical Methods in Natural Language Processing and the 9th International Joint Conference on Natural Language Processing, EMNLPIJCNLP' 19, pages 5636-5646, Hong Kong, China.

Leon Derczynski, Kalina Bontcheva, Maria Liakata, Rob Procter, Geraldine Wong Sak Hoi, and Arkaitz Zubiaga. 2017. SemEval-2017 Task 8: RumourEval: Determining rumour veracity and support for rumours. In Proceedings of the 11th International Workshop on Semantic Evaluation, SemEval '17, pages 60-67, Vancouver, Canada.

Dimitar Dimitrov, Bishr Bin Ali, Shaden Shaar, Firoj Alam, Fabrizio Silvestri, Hamed Firooz, Preslav Nakov, and Giovanni Da San Martino. 2021a. Detecting propaganda techniques in memes. In Proceedings of the Joint Conference of the 59th Annual Meeting of the Association for Computational Linguistics and the 11th International Joint Conference on Natural Language Processing, ACL-IJCNLP' 21, pages 6603-6617.

Dimiter Dimitrov, Bishr Bin Ali, Shaden Shaar, Firoj Alam, Fabrizio Silvestri, Hamed Firooz, Preslav Nakov, and Giovanni Da San Martino. 2021b. Task 6 at SemEval-2021: Detection of persuasion techniques in texts and images. In Proceedings of the 15th International Workshop on Semantic Evaluation, SemEval '21, pages 70-98.

Yoan Dinkov, Ivan Koychev, and Preslav Nakov. 2019 Detecting toxicity in news articles: Application to Bulgarian. In Proceedings of the International Conference on Recent Advances in Natural Language Processing, RANLP '19, pages 247-258, Varna, Bulgaria.

Tamer Elsayed, Preslav Nakov, Alberto BarrónCedeño, Maram Hasanain, Reem Suwaileh, Pepa Atanasova, and Giovanni Da San Martino. 2019. CheckThat! at CLEF 2019: Automatic identification and verification of claims. In Proceedings of the 41 st European Conference on Information Retrieval, ECIR '19, pages 309-315, Cologne, Germany.

Robert M Entman. 1993. Framing: Towards clarification of a fractured paradigm. McQuail's reader in mass communication theory, pages 390-397.
Emilio Ferrara, Onur Varol, Clayton Davis, Filippo Menczer, and Alessandro Flammini. 2016. The rise of social bots. Commun. ACM, 59(7):96-104.

Pepa Gencheva, Preslav Nakov, Lluís Màrquez, Alberto Barrón-Cedeño, and Ivan Koychev. 2017. A context-aware approach for detecting worthchecking claims in political debates. In Proceedings of the International Conference on Recent Advances in Natural Language Processing, RANLP' 17, pages 267-276, Varna, Bulgaria.

Genevieve Gorrell, Elena Kochkina, Maria Liakata, Ahmet Aker, Arkaitz Zubiaga, Kalina Bontcheva, and Leon Derczynski. 2019. SemEval-2019 task 7: RumourEval, determining rumour veracity and support for rumours. In Proceedings of the 13th International Workshop on Semantic Evaluation, SemEval '19, pages 845-854, Minneapolis, MN, USA.

Ivan Habernal, Raffael Hannemann, Christian Pollak, Christopher Klamm, Patrick Pauli, and Iryna Gurevych. 2017. Argotario: Computational argumentation meets serious games. In Proceedings of the 2017 Conference on Empirical Methods in Natural Language Processing: System Demonstrations, EMNLP'17, pages 7-12, Copenhagen, Denmark.

Ivan Habernal, Patrick Pauli, and Iryna Gurevych 2018. Adapting serious game for fallacious argumentation to German: Pitfalls, insights, and best practices. In Proceedings of the 11th International Conference on Language Resources and Evaluation, LREC '18, pages 3329-3335, Miyazaki, Japan.

Fatima Haouari, Maram Hasanain, Reem Suwaileh, and Tamer Elsayed. 2021. ArCOV-19: The first Arabic COVID-19 Twitter dataset with propagation networks. In Proceedings of the Sixth Arabic Natural Language Processing Workshop, ANLP '21, pages 82-91.

Momchil Hardalov, Arnav Arora, Preslav Nakov, and Isabelle Augenstein. 2021. A survey on stance detection for mis- and disinformation identification. arXiv/2103.00242.

Momchil Hardalov, Ivan Koychev, and Preslav Nakov. 2016. In search of credible news. In Proceedings of the 17th International Conference on Artificial Intelligence: Methodology, Systems, and Applications, AIMSA '16, pages 172-180, Varna, Bulgaria.

Naeemul Hassan, Chengkai Li, and Mark Tremayne. 2015. Detecting check-worthy factual claims in presidential debates. In Proceedings of the 24th ACM International Conference on Information and Knowledge Management, CIKM '15, pages 18351838, Melbourne, Australia.

Tamanna Hossain, Robert L. Logan IV, Arjuna Ugarte, Yoshitomo Matsubara, Sean Young, and Sameer Singh. 2020. COVIDLies: Detecting COVID-19 misinformation on social media. In Proceedings of the 1st Workshop on NLP for COVID-19 (Part 2) at EMNLP. 
Israa Jaradat, Pepa Gencheva, Alberto Barrón-Cedeño, Lluís Màrquez, and Preslav Nakov. 2018. ClaimRank: Detecting check-worthy claims in Arabic and English. In Proceedings of the 16th Annual Conference of the North American Chapter of the Association for Computational Linguistics, NAACLHLT '18, pages 26-30, New Orleans, LA, USA.

Dilek Küçük and Fazli Can. 2020. Stance detection: A survey. ACM Comput. Surv., 53(1).

Haewoon Kwak, Jisun An, and Yong-Yeol Ahn. 2020. A systematic media frame analysis of 1.5 million New York Times articles from 2000 to 2017. In Proceedings of the 12th ACM Conference on Web Science, WebSci '20, pages 305-314, Southampton, United Kingdom.

J Richard Landis and Gary G Koch. 1977. The measurement of observer agreement for categorical data. biometrics, pages 159-174.

David M.J. Lazer, Matthew A. Baum, Yochai Benkler, Adam J. Berinsky, Kelly M. Greenhill, Filippo Menczer, Miriam J. Metzger, Brendan Nyhan, Gordon Pennycook, David Rothschild, Michael Schudson, Steven A. Sloman, Cass R. Sunstein, Emily A. Thorson, Duncan J. Watts, and Jonathan L. Zittrain. 2018. The science of fake news. Science, 359(6380):1094-1096.

Lifang Li, Qingpeng Zhang, Xiao Wang, Jun Zhang, Tao Wang, Tian-Lu Gao, Wei Duan, Kelvin Kamfai Tsoi, and Fei-Yue Wang. 2020. Characterizing the propagation of situational information in social media during COVID-19 epidemic: A case study on Weibo. IEEE Transactions on Computational Social Systems, 7(2):556-562.

Yaliang Li, Jing Gao, Chuishi Meng, Qi Li, Lu Su, Bo Zhao, Wei Fan, and Jiawei Han. 2016. A survey on truth discovery. SIGKDD Explor. Newsl., 17(2):1-16.

Siyi Liu, Lei Guo, Kate Mays, Margrit Betke, and Derry Tanti Wijaya. 2019. Detecting frames in news headlines and its application to analyzing news framing trends surrounding US gun violence. In Proceedings of the 23rd Conference on Computational Natural Language Learning, CoNLL' 19, pages 504-514, Hong Kong, China.

Richard J Medford, Sameh N Saleh, Andrew Sumarsono, Trish M Perl, and Christoph U Lehmann. 2020 An "Infodemic": Leveraging high-volume Twitter data to understand early public sentiment for the coronavirus disease 2019 outbreak. OFID, 7(7).

Tsvetomila Mihaylova, Georgi Karadzhov, Pepa Atanasova, Ramy Baly, Mitra Mohtarami, and Preslav Nakov. 2019. SemEval-2019 task 8: Fact checking in community question answering forums. In Proceedings of the 13th International Workshop on Semantic Evaluation, SemEval '19, pages 860869, Minneapolis, MN, USA.
Tsvetomila Mihaylova, Preslav Nakov, Lluís Màrquez, Alberto Barrón-Cedeño, Mitra Mohtarami, Georgi Karadjov, and James Glass. 2018. Fact checking in community forums. In Proceedings of the ThirtySecond AAAI Conference on Artificial Intelligence, AAAI '18, pages 879-886, New Orleans, LA, USA.

Preslav Nakov, Firoj Alam, Shaden Shaar, Giovanni Da San Martino, and Yifan Zhang. 2021a. A second pandemic? Analysis of fake news about COVID19 vaccines in Qatar. In Proceedings of the International Conference on Recent Advances in Natural Language Processing, RANLP' 21.

Preslav Nakov, Alberto Barrón-Cedeño, Tamer Elsayed, Reem Suwaileh, Lluís Màrquez, Wajdi Zaghouani, Pepa Atanasova, Spas Kyuchukov, and Giovanni Da San Martino. 2018. Overview of the CLEF-2018 CheckThat! Lab on automatic identification and verification of political claims. In Proceedings of the Ninth International Conference of the CLEF Association: Experimental IR Meets Multilinguality, Multimodality, and Interaction, Lecture Notes in Computer Science, pages 372-387, Avignon, France.

Preslav Nakov, David Corney, Maram Hasanain, Firoj Alam, Tamer Elsayed, Alberto Barrón-Cedeño, Paolo Papotti, Shaden Shaar, and Giovanni Da San Martino. 2021b. Automated fact-checking for assisting human fact-checkers. In Proceedings of the 30th International Joint Conference on Artificial Intelligence, IJCAI '21, pages 4551-4558.

Preslav Nakov, Giovanni Da San Martino, Tamer Elsayed, Alberto Barrón-Cedeño, Rubén Míguez, Shaden Shaar, Firoj Alam, Fatima Haouari, Maram Hasanain, Nikolay Babulkov, Alex Nikolov, Gautam Kishore Shahi, Julia Maria Struß, and Thomas Mandl. 2021c. The CLEF-2021 CheckThat! lab on detecting check-worthy claims, previously factchecked claims, and fake news. In Proceedings of the 43rd European Conference on Information Retrieval, ECIR '21, pages 639-649.

Preslav Nakov, Husrev Taha Sencar, Jisun An, and Haewoon Kwak. 2021d. A survey on predicting the factuality and the bias of news media. $\operatorname{arXiv/2103.12506.~}$

Van-Hoang Nguyen, Kazunari Sugiyama, Preslav Nakov, and Min-Yen Kan. 2020. FANG: Leveraging social context for fake news detection using graph representation. In Proceedings of the 29th ACM International Conference on Information and Knowledge Management, CIKM '20, pages 1165-1174.

Ayush Patwari, Dan Goldwasser, and Saurabh Bagchi. 2017. TATHYA: a multi-classifier system for detecting check-worthy statements in political debates. In Proceedings of the 2017 ACM on Conference on Information and Knowledge Management, CIKM '17, pages 2259-2262, Singapore. 
Cristina M Pulido, Beatriz Villarejo-Carballido, Gisela Redondo-Sama, and Aitor Gómez. 2020. COVID19 infodemic: More retweets for science-based information on coronavirus than for false information. International Sociology, 35(4):377-392.

Hannah Rashkin, Eunsol Choi, Jin Yea Jang, Svitlana Volkova, and Yejin Choi. 2017. Truth of varying shades: Analyzing language in fake news and political fact-checking. In Proceedings of the Conference on Empirical Methods in Natural Language Processing, EMNLP' 17, pages 2931-2937, Copenhagen, Denmark.

Shaden Shaar, Firoj Alam, Giovanni Da San Martino, and Preslav Nakov. 2021a. The role of context in detecting previously fact-checked claims. Arxiv/2104.07423.

Shaden Shaar, Firoj Alam, Giovanni Da San Martino, Alex Nikolov, Wajdi Zaghouani, Preslav Nakov, and Anna Feldman. 2021b. Findings of the NLP4IF2021 shared tasks on fighting the COVID-19 infodemic and censorship detection. In Proceedings of the Fourth Workshop on NLP for Internet Freedom: Censorship, Disinformation, and Propaganda, pages 82-92.

Shaden Shaar, Maram Hasanain, Bayan Hamdan, Zien Sheikh Ali, Fatima Haouari, Alex Nikolov, Mucahid Kutlu, Yavuz Selim Kartal, Firoj Alam, Giovanni Da San Martino, Alberto Barrón-Cedeño, Rubén Míguez, Javier Beltrán, Tamer Elsayed, and Preslav Nakov. 2021c. Overview of the CLEF-2021 CheckThat! lab task 1 on check-worthiness estimation in tweets and political debates. In Working Notes of CLEF 2021-Conference and Labs of the Evaluation Forum, CLEF' 2021. CEUR-WS.org.

Shaden Shaar, Alex Nikolov, Nikolay Babulkov, Firoj Alam, Alberto Barrón-Cedeño, Tamer Elsayed, Maram Hasanain, Reem Suwaileh, Fatima Haouari, Giovanni Da San Martino, and Preslav Nakov. 2020. Overview of CheckThat! 2020 English: Automatic identification and verification of claims in social media. In Working Notes of CLEF 2020-Conference and Labs of the Evaluation Forum, CEUR Workshop Proceedings. CEUR-WS.org.

Kai Shu, Amy Sliva, Suhang Wang, Jiliang Tang, and Huan Liu. 2017. Fake news detection on social media: A data mining perspective. SIGKDD Explor. Newsl., 19(1):22-36.

Xingyi Song, Johann Petrak, Ye Jiang, Iknoor Singh, Diana Maynard, and Kalina Bontcheva. 2021. Classification aware neural topic model for COVID-19 disinformation categorisation. PLOS ONE, 16(2).

James Thorne and Andreas Vlachos. 2018. Automated fact checking: Task formulations, methods and future directions. In Proceedings of the 27th International Conference on Computational Linguistics, COLING '18, pages 3346-3359, Santa Fe, NM, USA.
Slavena Vasileva, Pepa Atanasova, Lluís Màrquez, Alberto Barrón-Cedeño, and Preslav Nakov. 2019. It takes nine to smell a rat: Neural multi-task learning for check-worthiness prediction. In Proceedings of the International Conference on Recent Advances in Natural Language Processing, RANLP '19, pages 1229-1239, Varna, Bulgaria.

Bertie Vidgen, Scott Hale, Ella Guest, Helen Margetts, David Broniatowski, Zeerak Waseem, Austin Botelho, Matthew Hall, and Rebekah Tromble. 2020. Detecting East Asian prejudice on social media. In Proceedings of the Workshop on Online Abuse and Harms, WOAH '20, pages 162-172.

Soroush Vosoughi, Deb Roy, and Sinan Aral. 2018. The spread of true and false news online. Science, 359(6380):1146-1151.

William Yang Wang. 2017. "Liar, liar pants on fire": A new benchmark dataset for fake news detection. In Proceedings of the 55th Annual Meeting of the Association for Computational Linguistics, ACL '17, pages 422-426, Vancouver, Canada.

Samuel C Woolley and Philip N Howard. 2018. Computational propaganda: political parties, politicians, and political manipulation on social media. Oxford University Press.

Seunghak Yu, Giovanni Da San Martino, Mitra Mohtarami, James Glass, and Preslav Nakov. 2021. Interpretable propaganda detection in news articles. In Proceedings of the International Conference on Recent Advances in Natural Language Processing, RANLP'21.

Savvas Zannettou, Michael Sirivianos, Jeremy Blackburn, and Nicolas Kourtellis. 2019. The web of false information: Rumors, fake news, hoaxes, clickbait, and various other shenanigans. J. Data and Information Quality, 11(3):10:1-10:37.

Yifan Zhang, Giovanni Da San Martino, Alberto Barrón-Cedeño, Salvatore Romeo, Jisun An, Haewoon Kwak, Todor Staykovski, Israa Jaradat, Georgi Karadzhov, Ramy Baly, Kareem Darwish, James Glass, and Preslav Nakov. 2019. Tanbih: Get to know what you are reading. In Proceedings of the Conference on Empirical Methods in Natural Language Processing and the 9th International Joint Conference on Natural Language Processing: System Demonstrations, EMNLP-IJCNLP '19, pages 223-228, Hong Kong, China.

Xinyi Zhou, Apurva Mulay, Emilio Ferrara, and Reza Zafarani. 2020. ReCOVery: A multimodal repository for COVID-19 news credibility research. In Proceedings of the 29th ACM International Conference on Information and Knowledge Management, CIKM'20, pages 3205-3212.

Arkaitz Zubiaga, Ahmet Aker, Kalina Bontcheva, Maria Liakata, and Rob Procter. 2018. Detection and resolution of rumours in social media: A survey. ACM Comput. Surv., 51(2). 